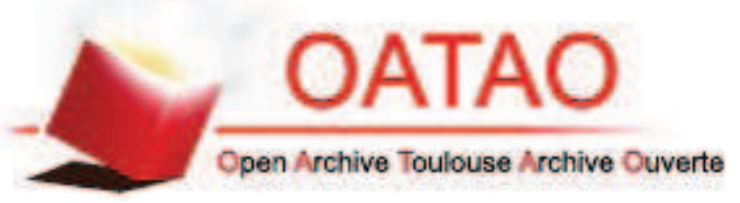

OATAO is an open access repository that collects the work of Toulouse researchers and makes it freely available over the web where possible.

This is an author-deposited version published in : http://oatao.univ-toulouse.fr/ Eprints ID : 10531

To link to this article : DOI: 10.1016/j.ces.2012.03.011

http://dx.doi.org/10.1016/j.ces.2012.03.011

To cite this version : Haroun, Yacine and Raynal, Ludovic and Legendre, Dominique Mass transfer and liquid hold-up determination in structured packing by CFD. (2012) Chemical Engineering Science, vol. 75 . pp. 342348. ISSN 0009-2509

Any correspondance concerning this service should be sent to the repository administrator: staff-oatao@ listes-diff.inp-toulouse.fr 


\title{
Mass transfer and liquid hold-up determination in structured packing by CFD
}

\author{
Y. Haroun ${ }^{\text {a,*, L. Raynal }}{ }^{\text {a }}$, D. Legendre ${ }^{\mathrm{b}, \mathrm{c}}$ \\ a IFP Energies nouvelles Rond-point de l'échangeur de Solaize, BP 3, 69360 Solaize, France \\ ${ }^{\mathrm{b}}$ Université de Toulouse, INPT, UPS, IMFT (Institut de Mécanique des Fluides de Toulouse), Allée Camille Soula, F-31400 Toulouse, France

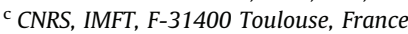

Keywords:

Mass transfer

Structured packing

Two-phase flow

CFD

$\mathrm{CO}_{2}$ capture

Gas treatment

\begin{abstract}
A B S T R A C T
Mass transfer and liquid hold-up in structured packing geometry are investigated using the volume of fluid method. Numerical simulations of two-dimensional co-current gas-liquid flow on structured packing with interfacial mass transfer are performed. The volume of fluid method is used to capture the gas-liquid interface motion. The mass transfer is computed by solving the concentration equation with an adapted modeling of the solubility (Haroun et al., 2010b). The liquid hold-up and the mass transfer are studied as function of liquid flow rate and structured packing geometry. Results show how the liquid flow rate and the complex geometry affect the liquid film flow topology and the interfacial mass transfer. For a specified packing geometry, it is demonstrated that for low liquid flow rate, the liquid film remains uniform and follow closely the profile of the structured wall. For uniform liquid film flow along packing wall, it is found that the liquid hold-up is in good agreement with the model proposed by Billet and Schultes (1999) and Raynal and Royon-Lebeaud (2007). When increasing the liquid flow rate, the liquid film does not follow the shape of the structured wall anymore, a static holdup (recirculation zone) form in the cavities and grows as the Reynolds number increases until covering most of the packing cavities. The present work gives the liquid hold-up evolution for each liquid film flow regime according to the Reynolds number and the dimensionless amplitude of the corrugation. Concerning the liquid side mass transfer, it is found that the liquid side mass transfer is well predicted by the Higbie (1935) theory provided that adequate velocity and length scales are considered for exposure time determination. The exposure time of fluid element at the interface corresponds to the ratio between the curvilinear distance between two periodic corrugation contact point and the interface velocity. An exposure time model is proposed taking into the account physical and geometric parameters.
\end{abstract}

\section{Introduction}

Structured packings are widely used in distillation and gas treatment processes and are good candidates for $\mathrm{CO}_{2}$ capture processes since they develop large effective area per unit volume ranging from 250 to $750 \mathrm{~m}^{2} / \mathrm{m}^{3}$, and large void fraction (porosity) around $90 \%$. The high geometric area and the high void fraction allow respectively to induce high mass transfer efficiency and low pressure drop. Although many investigations have been made (Billet, 1995; Bravo et al., 1985; Fair and Bravo, 1990), lot of understanding is still needed, in particular because the required experimental work is quite time consuming and does not easily allow to investigate the influence of all parameters on mass transfer and liquid hold-up, such as liquid viscosity, density or packing geometry. In this context the Computational Fluid

\footnotetext{
* Corresponding author. Tel.: +334377028 59; fax: +33437702008 E-mail address: yacine.haroun@ifpen.fr (Y. Haroun).
}

Dynamics (CFD) can be an interesting tool in addition to experimental works to increase the knowledge on the mass transfer and liquid hold-up in structured packings.

In recent years, several works can be found in the literature, which deal with the CFD simulations of multiphase flow in structured packings. Raynal et al. (2004) perform 2D CFD-simulations with the VOF method to determine the liquid thickness on a wavy plate similar to the packing surface and the results are used to derive the liquid hold-up on the structured packing. Ataki (2006) investigated the wetting in the structured packing elements using the volume of fluid methods and found a good agreement between the experimental and the CFD simulation results. Raynal and Royon-Lebeaud (2007) used the VOF method for gas-liquid flow simulation at small scale, and the results are further used at large scale in three-dimensional calculation with a geometry corresponding to complete packed columns. More recently, by considering thermodynamic equilibrium of chemical species' concentration at the interface, Haroun et al. (2010a) studied physical and reactive mass transfer in thin film flow 
down structured packing using the VOF method. For a given packing geometry, the mass transfer is found to be increasing compared to the transfer in the plane liquid film (Haroun et al., 2010a). For physical and reactive mass transfer respectively, they report a good agreement between simulation results, the Higbie theory and the Brian et al. (1961) solution, providing that adequate liquid velocity and long scale are considered.

In this paper, numerical simulations are used to improve the modeling of mass transfer and liquid hold-up in structured packings. For this purpose, numerical simulations of two-dimensional co-current gas-liquid flow on structured packings with interfacial mass transfer are performed. The computational method is based on the volume of fluid formulation validated in a previous study for the numerical simulation of mass transfer at a liquid interface (Haroun et al., 2010b). The chemical species concentration equation is solved coupled to the Navier-Stokes equations. The thermodynamic equilibrium of chemical species at the interface is considered using Henry's law. This method has been proved to be able to simulate mass transfer simultaneously with the evolution of deformed interface (Haroun et al., 2010a,b).

In the Section 2, the governing equations are described. Section 3 presents the problem formulations. The results are presented in Section 4.

\section{Governing equations}

The numerical approach is based on the volume of fluid (VOF) method validated and used in previous studies (Haroun et al., 2010a,b). The implemented VOF method consists in a Eulerian description of each phase on a fixed grid, the interface between the two phases being calculated using the transport equation of the local volume fraction of one phase (Scardovelli and Zaleski, 1999). The two fluids are assumed to be Newtonian and incompressible with no phase change. Under isothermal condition and in the absence of any surfactant the surface tension is constant and uniform at the interface between the two fluids. The NavierStokes equations are solved according the one-fluid formulation. The purpose of the one-fluid formulation is to describe the physical parameters for both phases using only one equation valid in the entire computational domain:

$\nabla U=0$

$\frac{\partial U}{\partial t}+U \nabla U=-\frac{1}{\rho} \nabla P+g+\frac{1}{\rho} \nabla T-\frac{\sigma}{\rho}(\nabla n) n \delta_{I}$

where $U, P, \rho, g, \sigma$ and $T$ are the local velocity, pressure, density, gravity, surface tension and viscous deformation tensor respectively. The surface delta function $\delta_{I}$ is zero outside the interface, $n$ is the unit normal. The location of each phase is given by a scalar $F$ (called volume fraction or color function) that obeys to the transport equation:

$\frac{\partial F}{\partial t}+(V \nabla) F=0$

where $V$ is the interface velocity. As discussed in Haroun et al. (2010b), by arbitrary choice, the value of the volume fraction function $F$ is $F=1$ (respectively $F=0$ ) in the liquid (respectively gas). The local density and viscosity are calculated using a linear interpolation as

$\mu=\mu_{L} F+\mu_{G}(1-F)$

$\rho=\rho_{L} F+\rho_{G}(1-F)$

where the subscripts $G$ and $L$ refer to the gas and liquid respectively. For the calculation of capillary force, a continuum method (CSF) is employed. Proposed by Brackbill et al. (1992), this technique interprets a surface tension forces as a continuous volume forces. The Navier-Stokes equations are solved by using the JADIM code developed at IMFT (Legendre and Magnaudet, 1998).

The momentum equations are discretized on an orthogonal grid using a finite volume approach. The spatial discretization is performed using second-order centered differences. Timeadvancement is achieved through a third-order Runge-Kutta algorithm for advective and source terms and a Crank-Nicolson algorithm for viscous stresses. Incompressibility is satisfied at the end of each time step through a projection method. The overall algorithm is second-order accurate in both time and space. More detail on numerical algorithm used and the transport strategy of volume fraction may be found in Legendre and Magnaudet (1998) and Bonometti and Magnaudet (2007).

The VOF chemical species conservation equation for chemical species $j$ describing mass transfer by convection, diffusion, is defined in conservation form as (Haroun et al., 2010b):

$\frac{\partial C_{j}}{\partial t}+\nabla\left(U C_{j}\right)=\nabla\left(D_{j} \nabla C_{j}+\Phi_{j}\right)+W_{j}$

with

$\Phi_{j}=-\left(D_{j} \frac{C_{j}\left(1-H e_{j}\right)}{F+H e_{j}(1-F)} \nabla F\right)$

where $D_{j}$ is the molecular diffusivity, $W_{j}$ is the production term related to the chemical reaction and $J_{j}$ is the molecular flux given by Fick's law $J_{j}=-D_{j} \nabla C_{j}$. Expression (7) is required in the VOF formulation in order to respect the solubility of chemical species at the interface described by Henry's law:

$C_{G, j}^{i}=H e_{j} C_{L, j}^{i}$

and the continuity of the fluxes at the interface:

$\sum_{L, G} J_{j} n \delta_{I}=0$

$C_{G, j}^{i}$ and $C_{L, j}^{i}$ are the concentrations of chemical species at interface in the gas $(G)$ and in the liquid $(L)$ phase, and He is Henry's constant. Expression (7) allows for interpreting the jump conditions as continuous effects only active in the interface zone, where gradients of the volume of fluid fraction $F$ is present. So, the solubility law (Henry's law) is converted into a solubility flux. In the following, the subscript $j$ is suppressed to simplify the notation.

The local diffusivity is calculated using the harmonic interpolation (Eq. (10)). This interpolation derives from subcell discretization. It has been proved by Haroun et al. (2010b) to be more precise than linear interpolation:

$D=\frac{D_{L} D_{G}}{D_{G} F+D_{L}(1-F)}$

Several test cases were performed to validate chemical species equation resolution. Typical cases of validation are for example mass transfer in a falling liquid film, mass transfer between bubble and surrounded liquid phase, mass transfer in stagnant two-phase mixture. These validations may be found in Haroun et al. (2010b) and Haroun (2008).

The local liquid mass transfer coefficient $k_{L, \text { local }}$ is calculated from the gradient of concentration $C$ and flux of solubility as

$k_{L, \text { local }}=-\frac{(D \nabla C+\Phi) n_{L}}{\Delta C_{L, \text { local }}}$

where $n_{L}$ is the normal to the interface pointing into the liquid, given by $n_{L}=\nabla F /|\nabla F|$. The average mass transfer coefficient $k_{L}$ is 


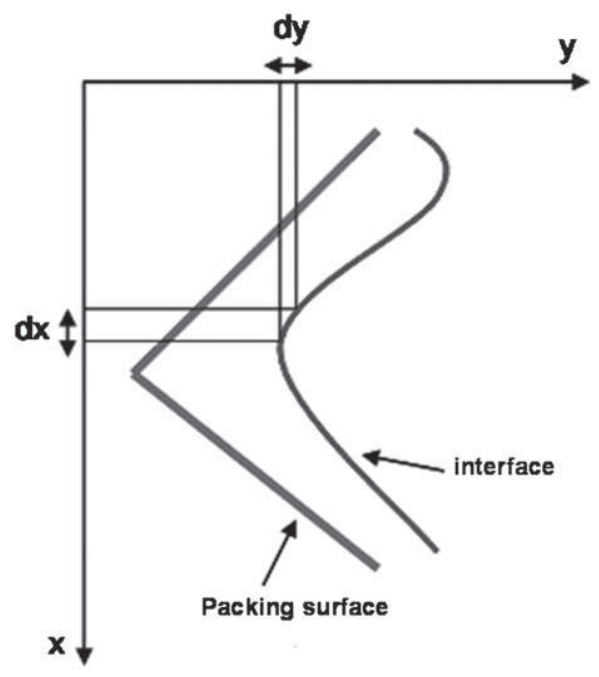

Fig. 1. Schematic illustration of parameters used to calculate total interface length.

defined as

$k_{L}=\frac{1}{\lambda} \int_{0}^{\lambda}-\frac{(D \nabla C+\Phi) n_{L}}{\Delta C_{L, \text { local }}} d \xi$

where $\lambda$ is the total interface length given by $\lambda=\sum^{n} \sqrt{d y^{2}+d x^{2}}$. $d y$ and $d x$ are a differential of the length measured numerically on the free surface as shown in Fig. 1. $\xi$ is the curvilinear coordinate associated to the interface ( $\xi=0$ at inlet).

$\Delta C_{L, \text { local }}$ is the local liquid concentration difference between the free surface region (at interface) and a reference concentration (for example the concentration in the bulk of the liquid phase). Note that the subscript local refers to local quantities.

\section{Problem formulation}

Structured packings are made of corrugating metal sheets arranged side by side with opposing channel orientation (Haroun et al., 2010a). Channels are oriented with angle from the horizontal that varies from $45^{\circ}$ to $60^{\circ}$. The structured packing performance depends on corrugation geometries and the angle from the horizontal direction. The $45^{\circ}$ angle is prevalent in the gas/liquid industrial contacting devices. The $60^{\circ}$ angle is designed for high capacity process.

The relevant geometrical characteristics of the structured packing are the angle of top vertex $\alpha$; the amplitude of the corrugation $H$ and the channel wavelength $X . \mathcal{L}$ denotes the packing wall path length traveled by the fluid between two periodic contact points of corrugation. The domain of study shown in Fig. 2a corresponds to a two dimension vertical cross section of the packing. A similar approach has been considered by Raynal et al. (2004) and Szulczewska et al. (2000) for simulation of gas-liquid flows in structured packing. The study is achieved with a constant geometry corresponding to $\alpha=90^{\circ}$.

Our objective is (i) to study the characteristics of liquid film falling under gravity and sheared by a co-current gas flow and (ii) to consider the transfer at the interface of a chemical species initially in the gas phase. The film is described using the local film thickness $e$. $\xi$ is the curvilinear coordinate associated to the interface (at inlet $\xi=0$ ). The total gas-liquid contact length in the domain of study corresponds to the interface length $\lambda$. The inlet gas flow rate is noted $q_{G}$. The liquid flow rate $q_{L}$ is injected with the inlet velocity $U o(y)$ and the inlet film thickness $\beta$ corresponding to the fully developed uniform thin film on a plane inclined wall (Nusselt, 1916):

$U o(y)=-\frac{U_{L, e f f}}{\beta^{2}} y(y-2 \beta)$

with $U_{L, e f f}=\frac{\rho_{L} g \sin (\alpha / 2) \beta^{2}}{2 \mu_{L}} ; \quad \beta=\left(\frac{3 v q_{L}}{g \sin (\alpha / 2)}\right)^{1 / 3}$

Free-slip boundaries are applied at the upper boundary and no-slip conditions are used at the wall (Fig. 2b). The dimensionless concentration $C^{*}=\left(C-C_{L, I}\right) /\left(C_{G, I}-C_{L, I}\right)=1$ is imposed for the gas at the inlet and at the upper boundary while $C^{*}=0$ is imposed for the inlet liquid. $C_{L, I}$ and $C_{G, I}$ are respectively the liquid and gas inlet concentrations. A Neumann boundary condition $\partial C / \partial x=0$ is used at the wall and the condition $\partial^{2} C / \partial x^{2}=0$ is imposed at the outlet boundary.

In the simulations presented here, we pay attention to the choice of the mesh resolution used to perform the simulations. The grid size is chosen so that the diffusion layer of concentration is correctly described. Some tests for plane liquid film or nonplane falling films have shown that the local flux at the interface becomes independent on the grid size if more than 4 cells are present in the concentration boundary layer (Haroun, 2008; Haroun et al., 2010b). Consequently, the meshes used to perform the following simulations have been built by respecting this criterium. An example of mesh used in this study is shown in Fig. 2b. It is a two-dimensional orthogonal grid mesh of 67,500 cells. A local refinement of mesh is imposed in the interface direction using a geometrical ratio to arrive at grid size of $0.1 \mu \mathrm{m}$ near the interface zone (Fig. $2 \mathrm{~b}$ ).

The results are discussed using non-dimensional numbers, namely the liquid Sherwood numbers, the liquid and gas Reynolds numbers and, the liquid and gas Schmidt numbers:

$S h_{L}=k_{L} L_{c h} / D_{L}, \quad \operatorname{Re}_{L}=4 q_{L} / v_{L}, \quad \operatorname{Re}_{G}=4 q_{G} / v_{G}, \quad S c_{L}=\mu_{L} / \rho_{L} D_{L}$, $S c_{G}=\mu_{G} / \rho_{G} D_{G}$

For industrial flow conditions observed in amine based $\mathrm{CO}_{2}$ capture process, the liquid load varies from approximately 50 to $250 \mathrm{~m}^{3} \mathrm{~m}^{-2} \mathrm{~h}^{-1}$. This corresponds to liquid film thickness varying from 0.3 to $0.4 \mathrm{~mm}$ and a liquid Reynolds number varying approximately from 100 to 400 . The liquid Schmidt number is much larger than unity, $S c_{L} \approx 500-600$, and is of order unity in gas, $S c_{L} \approx O(1)$. Thus the liquid Peclet number $P e_{L}=R e_{L} S c_{L}$ controlling the transfer at the interface is much larger than unity corresponding to thin concentration layer in the liquid. The diffusivity ratio is of order $D_{G} / D_{L} \approx O\left(10^{4}\right)$ and the Henry coefficient is $H e \approx 1.09$. Thus, in the present study, we consider cases that satisfy $R e_{L} \gg 1, P e_{L} \gg 1$ as well as we respect the gas/liquid properties ratio for the density, viscosity and the molecular diffusivity.

\section{Results and discussions}

\subsection{Film flow along corrugated wall}

Liquid film flow along periodic wall has been previously investigated by experimental and numerical studies (Vlachogiannis and Bontozoglou, 2002; Szulczewska et al., 2000; Raynal et al., 2004; Pozrikidis, 1988; Trifonov, 1998; Shetty and Cerro, 1997a,b; Valluri et al., 2005). These studies show that the relevant parameters are the liquid Reynolds number, the ratio between the liquid film thickness and the amplitude of the corrugation $\beta / X$, the ratio between the amplitude and the wavelength $H / X$ and the Bound number $B o=\rho_{L} g e^{2} / \sigma$ based on the interface thickness $e$. In the present study, we consider situation in which the wall is completely wetted and the surface tension have a negligible effect $(B o \gg 1)$. 
a

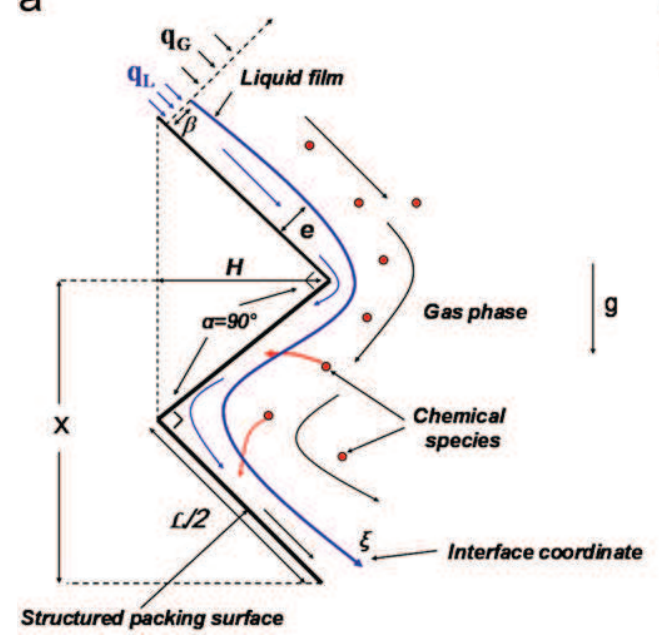

b

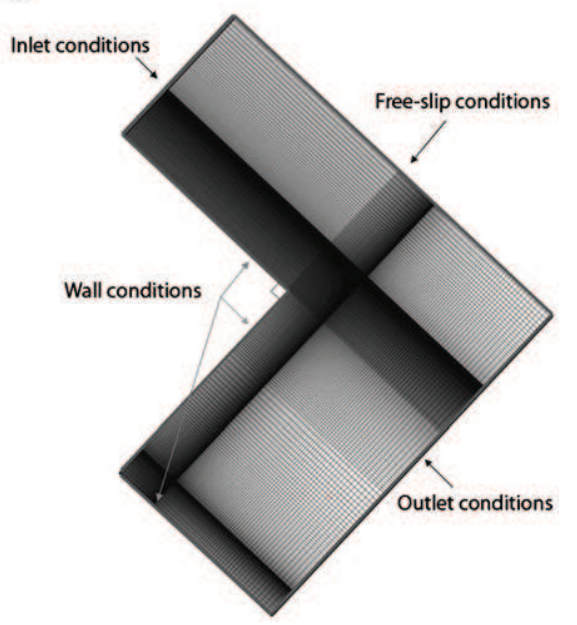

Fig. 2. (a) Schematic representation of computational domain and (b) mesh used illustration.

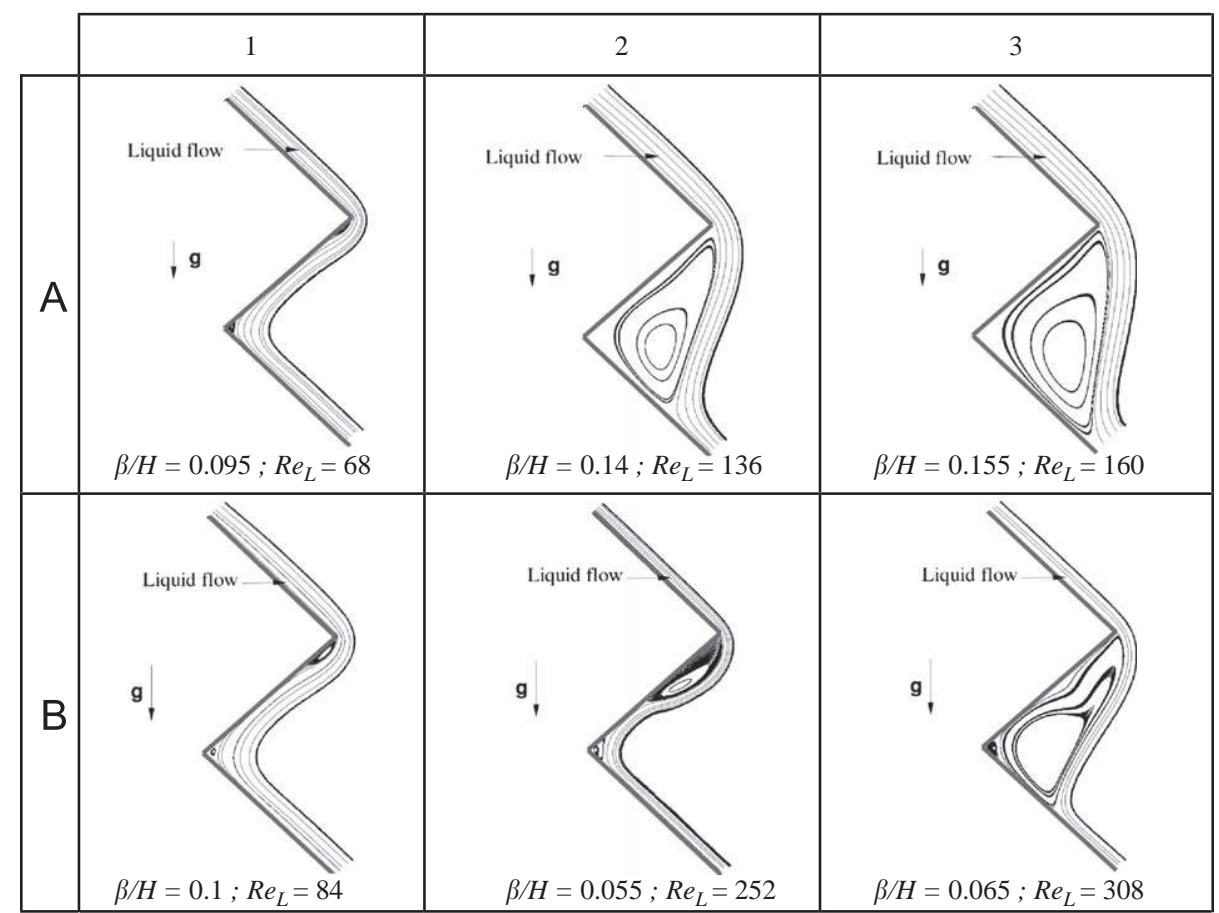

Fig. 3. Liquid film profile and stream lines obtained with the VOF simulations, for fixed ratio $H / X=0.5$ and gas Reynolds number $R e_{G}=180$.

In this section, we discuss the liquid film behavior by considering the effects of the normalized inlet film thickness $\beta / H$ and the liquid Reynolds number $R e_{L}$. Characteristic shapes of the liquid film are shown in Fig. 3 for different values of $\beta / H$ and $\operatorname{Re}_{L} . \beta$ is the inlet film thickness given by Eq. (13b). For low Reynolds number $R_{L}$ (i.e. low liquid flow rate), the liquid film follows closely the corrugate wall shape (Fig. 3A1). When increasing $\operatorname{Re}_{L}$ (i.e. the liquid flow rate), the thickness of the liquid film increases and a recirculation zone develops in the packing cavity. The recirculation zone grows as the liquid Reynolds number increases until the liquid fill completely the corrugation cavities as shown in Fig. 3(A2, A3). When the normalized liquid thickness $\beta / H$ is decreased (i.e. the corrugation amplitude $H$ is increased), Fig. 3(B1-B3) shows a similar behavior. However, the transition from the uniform liquid film to the liquid filling the corrugation cavities occurs at higher Reynolds numbers.
Based on visual observation, the liquid film shapes obtained from simulations are shown in the phase diagram $\left(H / \beta, \operatorname{Re}_{L}\right)$ indicated in Fig. 4. The cases corresponding to uniform film thickness are reported using filled symbol while open symbols are used for liquid film regime with filled corrugation cavities. Fig. 4 reveals that the transition can be described using a simple linear relation between the liquid Reynolds number $\operatorname{Re}_{L}$ and the ratio $H / \beta$ (dashed line in Fig. 4) given by

$\operatorname{Re}_{L} \approx 18.2 \frac{H}{\beta}$

For a given film thickness $\beta$, there is a critical liquid Reynolds number $R e_{L, C}$. When $R e<R e_{L, C}$, the liquid film is almost uniform along the corrugate wall while for $\operatorname{Re}_{L}>\operatorname{Re}_{L, C}$, the liquid fills the cavities of the corrugation. Thus relation (14) also indicates that 


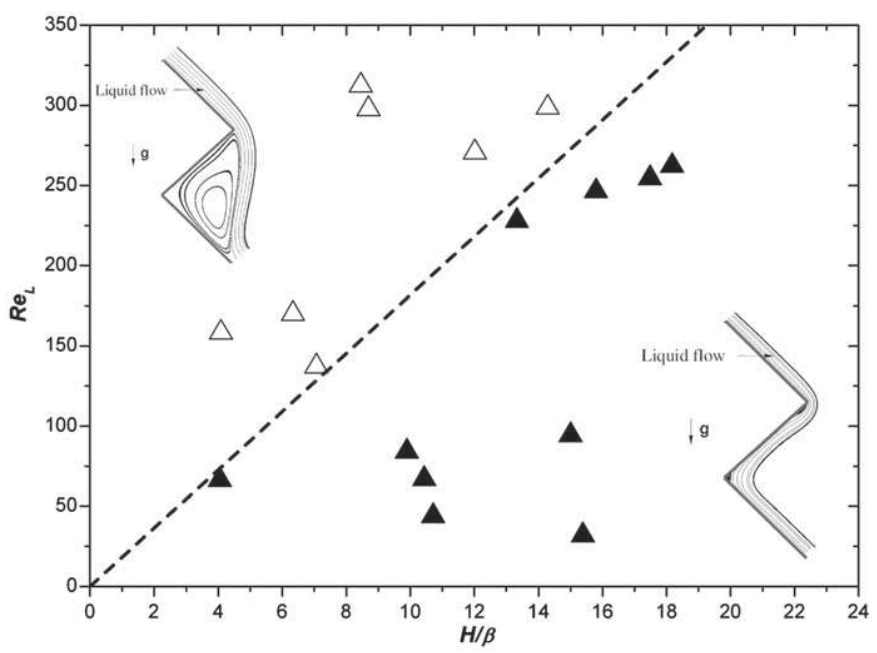

Fig. 4. Liquid film profiles as function of the liquid Reynolds number and the dimensionless amplitude of the corrugation $H / \beta$. (- -) Regime transition.

for a given liquid Reynolds number, there is a critical liquid thickness $\beta_{C}$ between the two film regimes.

A similar behavior is reported in recent studies, but no relation for the description of the transition is given. Raynal and RoyonLebeaud (2007) performed 2D CFD-simulations with the VOF method to determine the influence of the wall structure on the liquid hold-up for the Mellapak 250.Y geometry. They observe that depending on the liquid Reynolds number, recirculation zones form in the cavities of the roughness. The recirculation zone grows as the liquid Reynolds increases. They show higher liquid hold-up in the case of roughness wall compared to smooth walls by the presence of such recirculation zones in the cavities corrugations. With a similar technique Valluri et al. (2005) simulate water films flowing down over a doubly sinusoidal Mellapak 500.Y structured surface at $R e_{L}=10.45$ and 200. They found that for low flow rates, there is a small liquid accumulation in the valleys and the velocity show a parabolic profile within the film. At higher flow rates, a recirculation develops in the accumulation observed just below the macro-surface valley.

\subsection{Liquid hold-up}

Since the work of Bravo et al. (1985), the thickness of the liquid film has commonly been used to determine the liquid holdup $h_{L}$, using

$h_{L}=\langle e\rangle a_{g}$

where $\langle e\rangle$ is the average liquid film thickness in a periodic packing section equivalent to the wavelength $X . a_{g}\left(\mathrm{~m}^{2} \mathrm{~m}^{-3}\right)$ is the packing geometric area. Many models of liquid hold-up can be found in the literature. Duss et al. (1997), Olujic et al. (1999), Billet and Schultes (1999), and Raynal et al. (2004) model the liquid hold-up as the product between the thickness of the liquid film and the specific geometric area as

$\frac{h_{L}}{a_{g}}=\langle e\rangle=\beta$

where $\beta$ is the film thickness on a plane wall given by Eq. (13b). However, this relationship is valid only in the case of uniform thin film following the corrugate wall, thus valid for $\operatorname{Re}_{L}<R e_{L, C}$.

In Section 4.1, it has been shown that for $\operatorname{Re}_{L}>\operatorname{Re}_{L, C}$, the thickness of the liquid film grows in the packing section and a recirculation zone forms in the cavities. The presence of such recirculation increases the liquid hold-up. In this configuration,

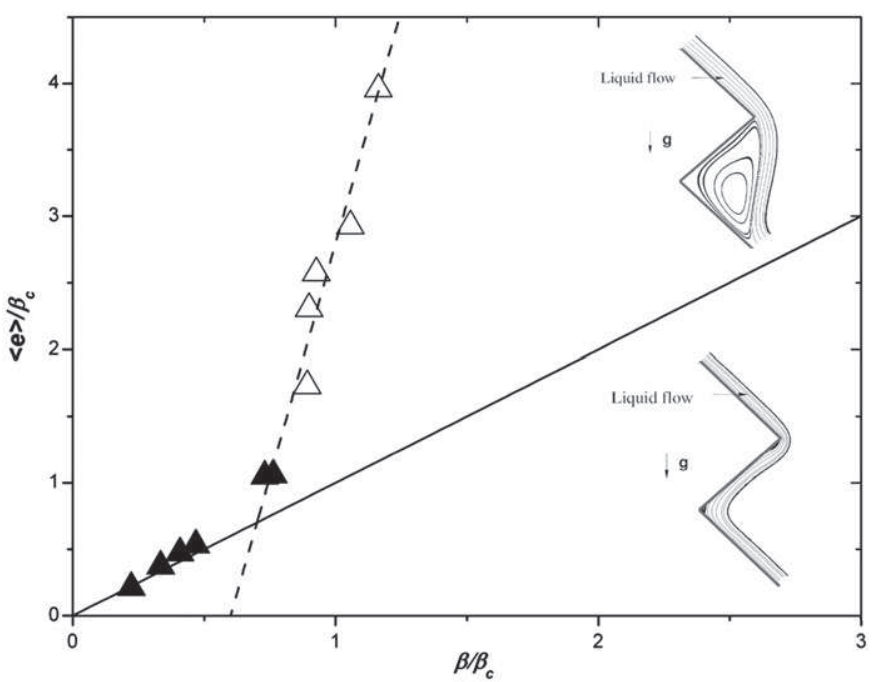

Fig. 5. Average liquid film thickness evolution in the periodic structured packing section. (- -) Eq. (17). (-) Eq. (16).

the liquid hold-up can be considered as the sum of a static holdup (recirculation zone) and a dynamic hold-up (Raynal and Royon-Lebeaud, 2007). In Fig. 5, the normalized average liquid film thickness $\left(\langle e\rangle \mid \beta_{C}\right)$ is compared to the liquid film model given by Eq. (16). The relation $\beta_{C}=18.2 H / R e_{L}$ is deduced from relation (14). Fig. 5 clearly shows that the evolution of the average film thickness follows two distinct behaviors. When the liquid film follows the corrugate wall (i.e. for $R_{L}<R e_{L, C}$ ), the average thickness film $\langle e\rangle$ is in good agreement with Eq. (16) and modeling commonly found in the literature to describe flow encountered in the structured packing columns. When the liquid flow rate increases (i.e. for $R e_{L}>R e_{L, C}$ ), we observe a clear change in the average film thickness behavior but the evolution remains linear. The slope is increased due to the presence of the static hold-up (recirculation zone) in the corrugation cavities. The expression of the average film thickness is found to be a good agreement with the following relation:

$\langle e\rangle=\frac{h_{L}}{a_{g}}=7 \beta-76.4 \frac{H}{R e_{L}}$

The intersection between the two film thickness curves permits to determine that the transition between the two behaviors occurs at $\operatorname{Re}_{L}=12.7 \mathrm{H} / \beta$. This value is lower than $\operatorname{Re}_{L, C}$ given by Eq. (14) because of the apparition of small recirculations below the picks and in the cavities of corrugation as clearly shown in Fig. 3B2. Note that Eq. (17) may overestimate the calculation of liquid hold-up in real operating conditions. Indeed, in our simulations the angle of the top of the corrugation is $90^{\circ}$. This angle is smaller than the angle traveled by the fluid in real flow condition. Since, a good agreement has been found with literature model when the liquid film is uniform, we can expect that Eq. (17) is an improved model for the recirculation regime. Moreover, our results outline the effect of the amplitude $H$ not considered in models from the literature.

\subsection{Mass transfer}

We consider in this section the interfacial mass transfer. Recently, Haroun et al. (2010a) have shown that for uniform thin film on a corrugated geometry, the transfer is larger than observed on a plane wall. They also reveal that the interfacial mass transfer is well predicted by the Higbie model $k_{L}=1.13 \sqrt{D / t_{\text {exp }}}$ if the interface velocity $U_{L, \text { eff }}$ and the interfacial 
exposure distance length $\lambda$ are used to calculate the exposure time, $t_{\exp }=\lambda / U_{L, e f f} . \lambda$ is the length traveled by the fluid between two periodic contact points of corrugation, and corresponds to the renewal interface distance in structured packing as discussed in Raynal et al. (2009),and Sidi-Boumedine and Raynal (2005).

Fig. 6 shows an example of the iso-concentration contour for a non-uniform film, when $R e>R e_{L, c}$. The figure shows that the recirculation zone in the accumulation region does not affect the $\mathrm{CO}_{2}$ concentration penetration in the liquid film. Indeed, the chemical species penetrate in a thin concentration boundary layer not affected by the recirculation. Due to the high value of the liquid Peclet number, the transfer is controlled by the advection at the interface. Therefore, for industrial operating condition $\left(P e_{L}>10^{4}\right)$, the liquid film can be considered as infinitely thick compared to the penetration distance of the chemical species. In

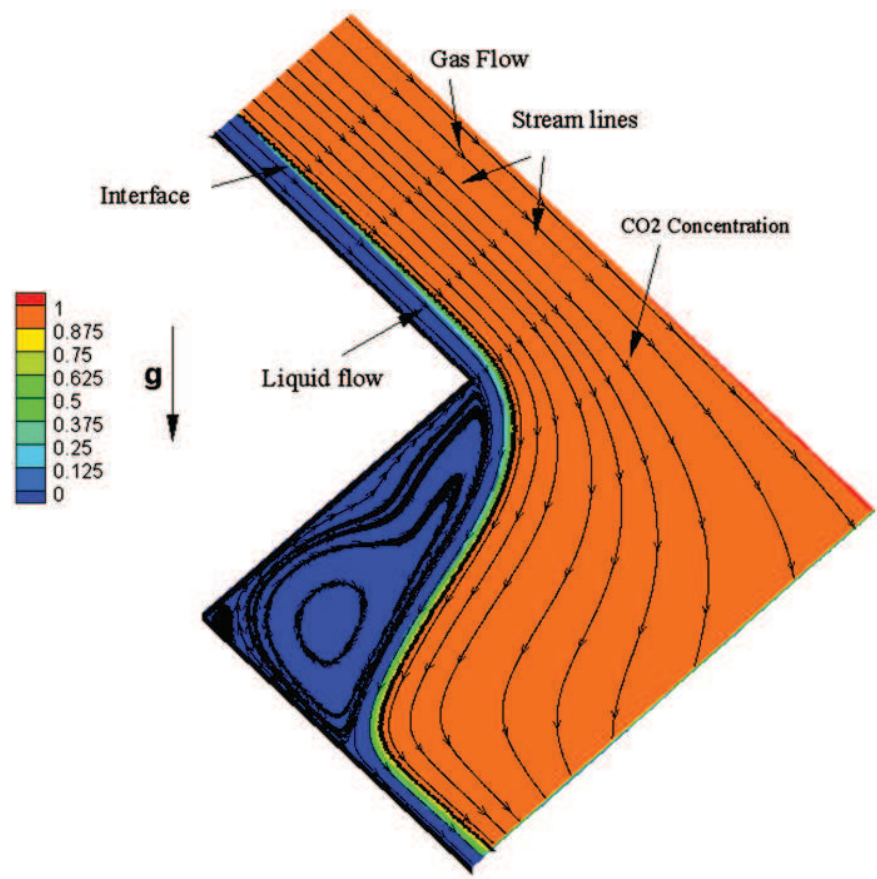

Fig. 6. Contour plot of the dimensionless $\mathrm{CO}_{2}$ concentration distribution at steady state. $\beta / H=0.065 ; R e_{L}=308 ; S c_{L}=5 ; P e_{L}=1540 ; R e_{G}=180, S c_{G}=0.6 ; H e=1.1$.

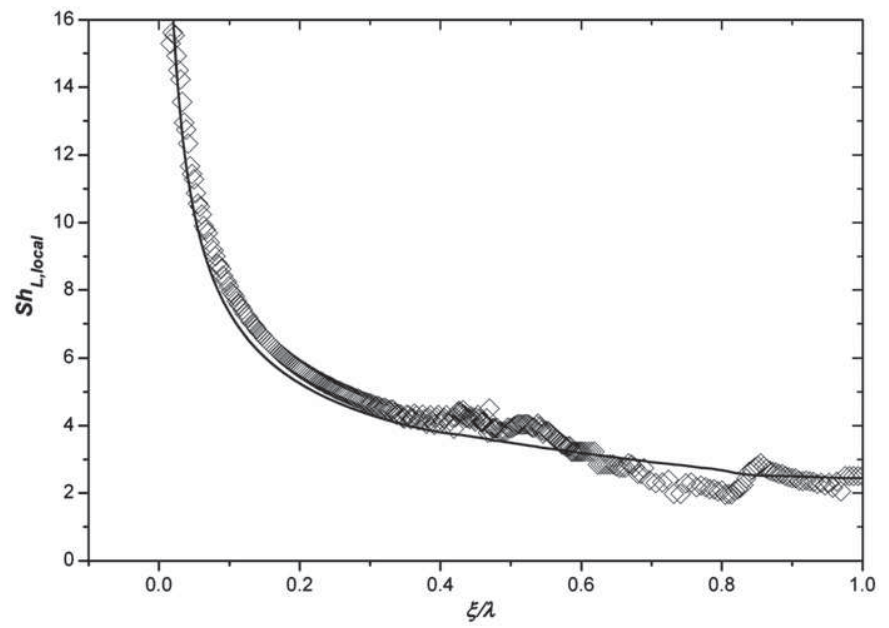

Fig. 7. Local evolution of the liquid side Sherwood number with the curvilinear interface distance from the inlet. Comparison between numerical results $(\diamond)$ and the Higbie model (-). $\beta / H=0.065 ; R e_{L}=308 ; S c_{L}=5 ; P e_{L}=1540 ; R e_{G}=180$, $S c_{G}=0.6 ; \mathrm{He}=1.1$.
Fig. 7, the corresponding local liquid Sherwood number is reported versus the curvilinear coordinate of the interface. The local transfer is compared with the penetration theory of Higbie where the local exposure time, $t_{\text {exp } \text {,local }}$ of fluid element at the interface corresponds to the ratio between the local curvilinear distance $\xi$ and the local velocity of the interface:

$t_{\text {exp,local }}=\xi / U_{L, \text { eff }}$

Despite the complex geometry considered and the presence of a significant recirculation (see Fig. 6) the agreement is very interesting. This confirms that the recirculation flow in the packing cavities does not affect the chemical species penetration. Consequently, the mass transfer is strongly dependent on the interface shape that controls the exposure time. For the geometry considered in this study, the exposure distance in a periodic section of packing has two evident limits, the length of the packing $\lambda=\mathcal{L}$ for very thin uniform liquid film and the vertical distance between two corrugations contact point $\lambda=X=\mathcal{L} / \sqrt{ } 2$ when the cavities is filled with the liquid.

Many models have been proposed in the literature in order to calculate the exposure time $t_{\text {exp }}$ in structured packings. Bravo et al. (1985), Fair and Bravo (1990) and Gualito et al. (1997) define the exposure time as the ratio between the corrugation spacing and the effective velocity based on the falling film solution for laminar flow. De Brito et al. (1992) computed the exposure time as function of the liquid hold-up, the corrugation spacing and the liquid load. More recently, for uniform thin liquid film, Raynal et al. (2009) defined the exposure time between two periodic contact point (corrugations peaks) as the ratio between the distance traveled by the fluid element and the velocity at the interface:

$t_{\text {exp }}^{*}=\mathcal{L} / U_{L, e f f}$

where $U_{L, \text { eff }}$ is given by Eq. (13b). Fig. 8 compares the exposure time $t_{C F D}$ deduced from our simulations with the above exposure time model $t_{\exp }^{*}$ proposed by Raynal et al. (2009). The figure shows that the simulated exposure time is located between $t_{C F D}=0.5 t_{\text {exp }}^{*}$ and $t_{C F D}=0.9 t_{\text {exp }}^{*}$. The exposure time is found to be always smaller than $t_{\text {exp }}^{*}$, since, the interfacial exposure distance is always smaller than the distance $\mathcal{L}$ as suggested by Raynal et al. (2009). The exposure time is also found to be larger than $t_{C F D}=0.5 t_{\text {exp }}^{*}$ corresponding to the limit of completely filled corrugation because the cavity is never completely filled in our simulation. Finally, the exposure time between two periodic corrugation

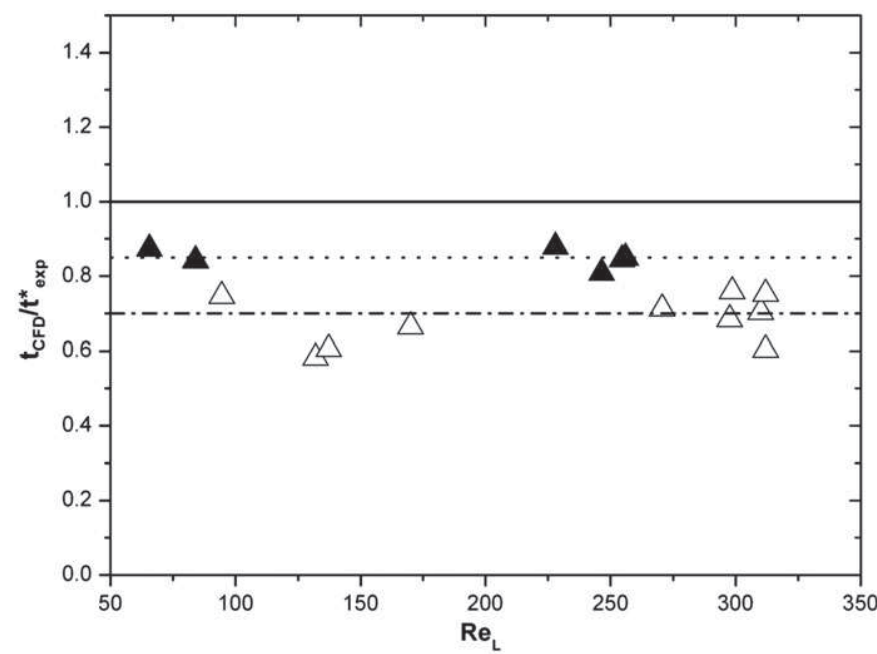

Fig. 8. Exposure time versus the liquid Reynolds number. Comparison between present work and (-) Raynal et al. (2008); (- -) present work: Eq. (18) for $K=0.85$; (- -) present work: Eq. (18) for $K=0.7$. 
contact points can be estimated using the following expression as shown in Fig. 8:

$t_{\text {exp }}=K \frac{L}{\left(\rho_{L} g \sin (\alpha / 2) \beta^{2} / 2 \mu_{L}\right)}$

where $K$ is shown to depend on the film regime: $K \approx 0.85$ for uniform film $\left(R e_{L}<R e_{L, C}\right)$ and $K \approx 0.7$ for film with recirculation $\left(R e_{L}>R e_{L, C}\right)$. This expression for the exposure time allows improving mass transfer determination by taking into account the variation of the film regime.

\section{Conclusion}

We have used direct numerical simulations to improve the understanding of the gas-liquid flow behavior in term of liquid hold-up and mass transfer modeling in structured packings. For low liquid flow rate, the liquid film has a low thickness and closely follows the corrugated wall shape. When increasing the liquid flow rate, the thickness of the liquid film becomes significant and a recirculation zone develops in the cavities. This recirculation zone grows as the Reynolds number increases until the liquid completely fills the corrugation. We show that the transition between these two regimes can be expressed in term of the liquid Reynolds number. The critical liquid Reynolds number evolves linearly with the ratio between corrugation amplitude and the inlet film thickness.

The liquid hold-up is found to significantly depend on the film shape. For uniform thin liquid film, the liquid hold-up is in good agreement with model found in the literature. When increasing the liquid flow rate, the recirculation zone (static liquid hold-up) that develops in corrugation cavities induces the increase of the liquid hold-up. A new predictive model has been proposed for this particular regime. Thus the amplitude of the corrugation is shown to be an important parameter to consider while it is not present in the models of the literature.

The mass transfer is not directly affected by the recirculation in the corrugation cavities. Indeed, the chemical species only penetrate in a very thin concentration layer so that the transfer process is controlled by the advection at the film interface. Our simulations show that the liquid side mass transfer is well predicted by the Higbie (1935) theory provided that the exposure time at the interface is determined using the curvilinear distance and the local velocity at the interface. Thus, the mass transfer is linked to the interface shape that controls the exposure time. Finally, a predictive model for the exposure time is proposed. In this study, the angle of the corrugation has been imposed to $90^{\circ}$. The effect of this angle has not yet been investigated. Such a study should be of great interest for improving models used for the design of efficient structured packing column.

\section{References}

Ataki, A., 2006. Wetting of Structured Packing Elements-CFD and Experiment. Ph.D. Thesis. Technical University of Kaiserslautern. Billet, R., 1995. Packed Towers. VCH, Weinheim, Germany.
Billet, R., Schultes, M., 1999. Prediction of mass transfer columns with dumped and arranged packings. Updated summary of the calculation method of Billet and Schultes. Chem. Eng. Res. Des. 77 (A6), 498-504.

Brian, P.L.T., Hurey, J.F., Hassettine, E.H., 1961. A.I.Ch.E. J. 7, 226

Bravo, J.L., Rocha, J.A., Fair, J.R., 1985. Mass transfer in gauze packing. Hydrocarbon Process., 91-95.

Brackbill, J.U., Khote, D.B., Zemach, C.A., 1992. A continuum method for modelling surface tension. J. Comput. Phys. 100, 335-354.

Bonometti, T., Magnaudet, J., 2007. An interface-capturing method for incompressible two-phase flows. Validation and application to bubble dynamics. Int. J. Multiphase Flow 33, 109-133.

Duss, M., Meierhofer, H., Bomio, P., 1997. Comparison between random and structured packings and a model to predict the efficiency of structured packing in distillation and absorption applications. In: Distillation and Absorption, Institute of Chemical Engineering Symposium Series no. 142, Maastricht, The Netherlands, vol. 1, pp. 439-452.

De Brito, H.M., von Stockar, U., Bomio, P., 1992 Predicting the liquid phase mass transfer coefficient $k_{L}$ for the Sulzer structured packing mellapack. In: Distillation and absorption, vol. 92, Institute of Chemical Engineering Symposium Series no. 128, Birmingham, UK, vol. 2, pp. B137-B144.

Fair, J.R., Bravo, J.L., 1990. Distillation columns containing structured packings. Chem. Eng. Prog., 19-29.

Gualito, J.J., Cerino, F.J., Cardenas, J.C., Rocha, J.A., 1997. Design method for distillation columns filled with metallic, ceramic, or plastic structured packings. Ind. Eng. Chem. Res. 36 (5), 1747-1757.

Haroun, Y., Legendre, D., Raynal, L., 2010a. Direct numerical simulation of reactive absorption in gas-liquid flow on structured packing using interface capturing method. Chem. Eng. Sci. 65, 351-356.

Haroun, Y., Legendre, D., Raynal, L., 2010b. Volume of fluid method for interfacial reactive mass transfer: application to stable liquid film. Chem. Eng. Sci. 65, 2896-2909.

Haroun, Y., 2008. Numerical Simulation Study of Gas/Liquid Reactive Mass Transfer Along Corrugated Sheets With Interface Tracking. Ph.D. Thesis. Institut National Polytechnique de Toulouse. 〈http://ethesis.inp-toulouse.fr/ archive/00000700/01/haroun.pdf $>$.

Higbie, R., 1935. The rate of absorption of a pure gas into a still liquid during short periods of exposure. Trans. A.I.Ch.E. 35, 365.

Legendre, D., Magnaudet, J., 1998. The lift force on a spherical bubble in a viscous linear shear flow. J. Fluid Mech. 368, 81-126.

Nusselt, W., 1916. Die Oberflachenkondensation des Wasserdampfes. Ver. Deut. Ingr. 60, 549.

Olujic, Z., Kamerbeek, A.B., de Graauw, J., 1999. A corrugation geometry based model for efficiency of structured distillation packing. Chem. Eng. Process. 38 (4-6), 683-695.

Pozrikidis, C., 1988. The flow of a liquid film along a periodic wall. J. Fluid Mech. $188,275-300$.

Raynal, L., Boyer, C., Ballaguet, J.P., 2004. Liquid hold-up and pressure drop determination in structured packing with CFD simulation. Can. J. Chem. Eng.

Raynal, L., Royon-Lebeaud, A., 2007. A multi-scale approach for CFD calculations of gas-liquid flow within large size column equipped with structured packing. Chem. Eng. Sci. 62, 7196-7204.

Raynal, L., Ben Rayana, F., Royon-Lebeaud, A., 2009. Use of CFD for $\mathrm{CO}_{2}$ absorbers optimum design: from local scale to large industrial scale. Energy Procedia.

Scardovelli, R. Zaleski, S, 1999. Direct numerical simulation of free-surface and interfacial flow. Annu. Rev. Fluid Mech. 31, 567-603.

Szulczewska B., Sek, J., Gorak, A., Zbicinski, I., 2000. CFD calculation of two-phase flow on elements of structured packing. In: Chisa International Conference, Praha, Czech Republic, August 27-37, Paper \#565, pp. 1-10.

Shetty, S.A. Cerro, R.L. 1997a. Estimation of liquid distribution and mass transfer parameters for ordered packings. In: Darton, R. (Ed.), Distillation and Absorption, Institute of Chemical Engineering Symposium Series no. 142 vol. 2, Maastricht, The Netherlands.

Shetty, S.A., Cerro, R.L., 1997b. Fundamental liquid flow correlations for the computation of design parameters for ordered packings. Ind. Eng. Chem. Res. 36 (3), 771-783.

Sidi-Boumedine, R., Raynal, L., 2005. Influence of the liquid hold-up in trickle bed reactors with structured packings. Catal. Today 105, 673-679.

Trifonov, Y., 1998. Viscous liquid film flows over a periodic surface. Int. J. Multiphase Flow 24 (7), 1139-1161.

Valluri, P., Matar, O.M., Hewitt, G.F., Mendes, M.A., 2005. Thin film flow over structured packings at moderate Reynolds numbers. Chem. Eng. Sci. 60 1965-1975.

Vlachogiannis, M., Bontozoglou, V., 2002. Experiments on laminar film flow along a periodic wall. J. Fluid Mech. 457. 